\title{
Jets and particle correlations in heavy ion collisions
}

\section{Matthew Nguyen*}

Laboratoire Leprince-Ringuet - École Polytechnique

E-mail: Matthew. Nguyen@ecern.ch

\section{on behalf of the LHC and RHIC collaborations}

\begin{abstract}
Measurements of jets and particle correlations in nucleus-nucleus collisions are intended to probe QCD interactions in the high temperature phase, where matter is understood to behave as a quarkgluon plasma. Two probes are reviewed: jets which are used to study the energy loss of hardscattered partons in this medium and particle correlations which are used to understand collective effects of the bulk matter. Whereas collisions of lighter systems, namely proton-ion and protonproton, initially served primarily as control experiments, certain (but not all) effects first observed in nucleus-nucleus collisions have proven to be pervasive in these systems. Comparative measurements in these three systems have broadened our understanding of many-body QCD phenomena, and raised new questions. This talk reviewed these recent developments.
\end{abstract}

The European Physical Society Conference on High Energy Physics

5-12 July, 2017

Venice

${ }^{*}$ Speaker. 
Jets and correlations are two topics of central importance in heavy-ion physics. They are related to paradigms that gained a foothold when data started to emerge from the RHIC program about a decade ago. Although jets were difficult to measure at RHIC, high $p_{\mathrm{T}}$ hadrons served as a proxy. In central, or highly overlapping, nucleus-nucleus (AA) collisions a large suppression of roughly a factor of 5 was observed, compared to the yield expected based on measurements in proton-proton (pp) collisions [1]. No such suppression was observed for peripheral AA collisions or for collisions of the same nucleus (gold) with the much lighter deuteron [2]. This data were taken as evidence of jet quenching, wherein a hard-scattered parton will lose energy in the quark-gluon plasma created in central AA collisions. The absence of such effects in light systems served as a control, showing that nuclear effects unrelated to quark-gluon plasma formation are not responsible for the large suppression, as such hot matter was not expected to be formed in these systems.

The second paradigm established at RHIC relates to the collectivity of the system produced. That collectivity is measured by particle correlations. In particular, the elliptic flow measures to what extent the initial asymmetry in non-central AA collisions propagates to an azimuthal anisotropy in the final state particles. At RHIC it was found that the measured elliptic flow is well-described by ideal hydrodynamics, i.e., viscous corrections are small [3,4]. In fact, the shear viscosity divided by the entropy comes remarkably close to the conjectured lower bound of $1 / 4 \pi$, which comes from string theory via the AdS / CFT correspondence [5].

\section{Jets in heavy ions: The advent of the LHC}

With the increased collision energy and hermetic detectors available at the LHC, it became possible to cleanly reconstruct jets in the heavy-ion environment for the first time. As in elementary particle collisions, jets reduce sensitivity to non-pertubative hadronization effects compared to hadron-based measurements. They also allow measurements of new observables, e.g., of the internal structure of jets, which turns out to be key for understanding the dynamics of parton energy loss.

A comparison of the nuclear modification factor $\left(R_{\mathrm{AA}}\right)$ of jets with that of charged hadrons turns out to be rather instructive. Charged particles can be measured precisely out to large $p_{\mathrm{T}}$ at the LHC, which gives some additional discrimination between models, as shown in Fig. 1 (left) for the $5 \%$ most central collisions at $\sqrt{s_{N N}}=5 \mathrm{TeV}$ [6]. Whereas, the charged hadron $R_{\mathrm{AA}}$ was fairly flat at RHIC, with the additional lever arm at the LHC, we observe that it grows with $p_{\mathrm{T}}$, eventually coming close to unity, which would indicate no modification of the yield compared to pp.

Turning to $R_{\mathrm{AA}}$ of jets, we see a somewhat different trend in Fig. 1 (right). The $R_{\mathrm{AA}}$ shows a slow rise, but is then remarkably flat out to nearly $1 \mathrm{TeV}$. This quenching is approximately independent of cone size out to $\mathrm{R}=0.4$, which indicates that the quenched energy is being deposited at large angle with respect to the jet axis. It's not immediately obvious how this suppression pattern is consistent with the charged hadron measurement, which seems to suggest that nuclear effects diminish at high $p_{\mathrm{T}}$. One possible explanation is that there's an interplay between the quenching and the fragmentation pattern of the jets, such that hard fragmenting jets are less quenched. A couple of examples of how such an effect might arise are discussed below.

Beyond single jets, dijets are also of interest as they carry equal $p_{\mathrm{T}}$ to leading order in elementary collisions. In AA collisions though, the two jets will not necessarily have the same path-length 

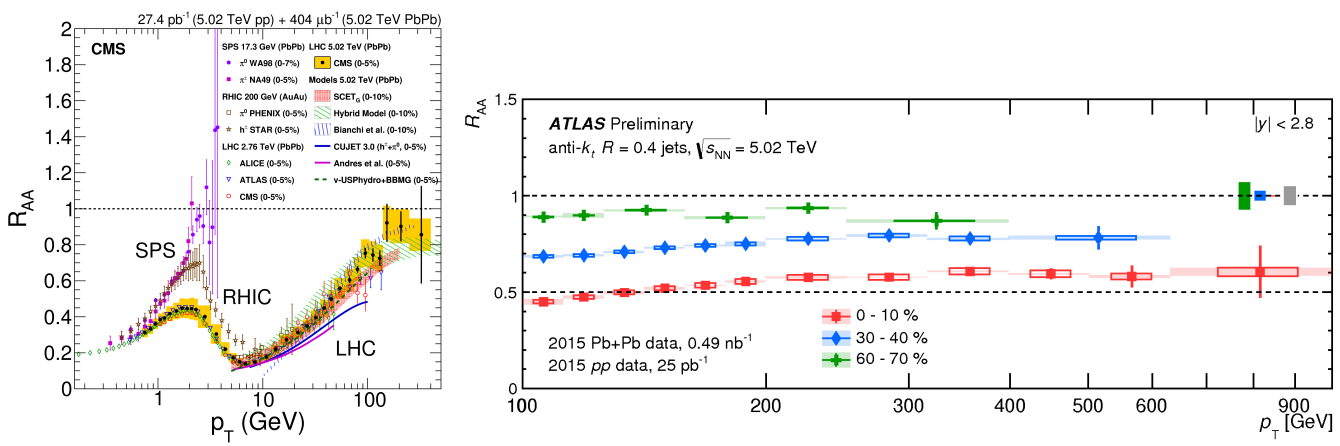

Figure 1: $R_{\mathrm{AA}}$ of charged hadrons (left) [6] and jets (right) [7] in central $\mathrm{PbPb}$ collisions at $5 \mathrm{TeV}$.

through the quark-gluon plasma. This gives the expectation that the medium produces an extra source of net imbalance between pairs of jets, an expectation that is borne out by the data, as shown in Fig. 2 (left). Although full event generators with jet quenching are still very much an emerging technology, it's interesting to see how they compare to this measurement. A comparison to JEWEL is shown, which is arguably the most advanced quenching generator available [8]. JEWEL manages to reproduce the trend of the data quite well. What is interesting about this particular calculation is that the imbalance is actually not driven by the path-length difference of the jets, which in this model is a sub-dominant effect. Instead the effect comes mostly from fluctuations in the fragmentation pattern of jets, which is exactly the sort of effect that could explain a difference in the jet and charged hadron $R_{\mathrm{AA}}$. It turns out that this effect emerges rather naturally in this model which implicitly takes into account the energy loss not only of the leading parton, as is done in most analytical calculations, but of the entire parton shower. Jets with a larger number of constituents tend to radiate more. Moreover, for the softer constituents energy tends to be pushed out of the jet cone via elastic collisions with the medium, a mechanism which is also included in this model.
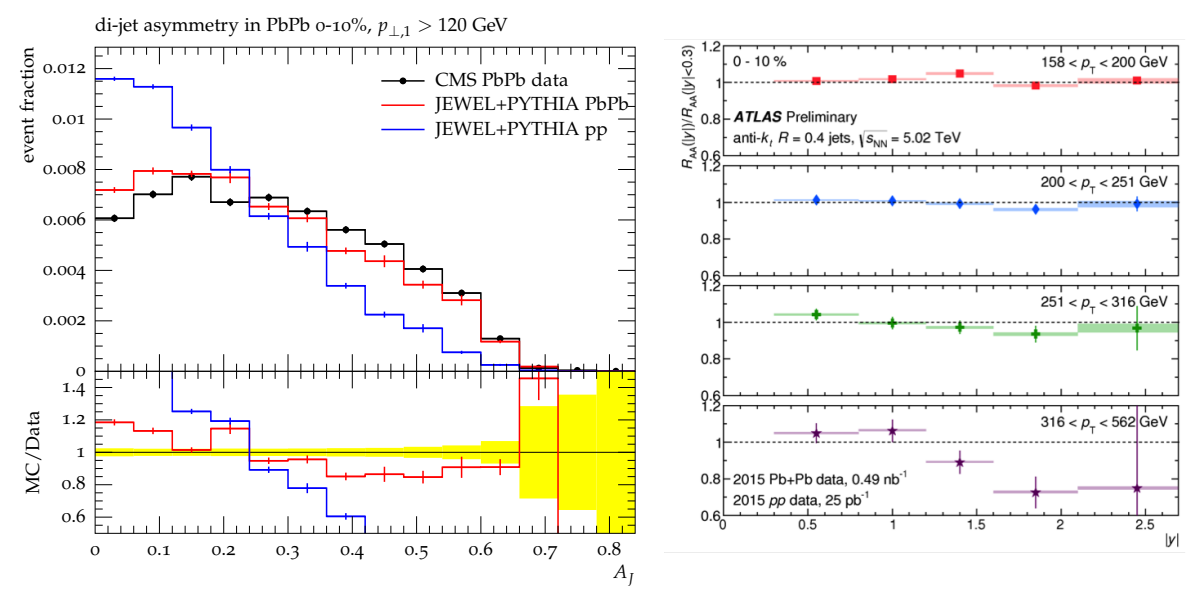

Figure 2: Left: dijet imbalance data [9] compared to JEWEL simulations [8]. Right: jet $R_{\mathrm{AA}}$ in bins of $y$ [7].

ATLAS recently published dijet imbalance results that are fully corrected for resolution effects for the first time [10]. After unfolding, a rather prominent feature emerges in the imbalance distribution at low $p_{\mathrm{T}}$, comparing AA to $\mathrm{pp}$, which gradually disappears at large $p_{\mathrm{T}}$. Going forward, it 
will be very interesting to see if any models can reproduce this feature.

Returning to jet $R_{\mathrm{AA}}$, another interesting feature emerges when we look differentially not only in $p_{\mathrm{T}}$, but also in rapidity. Figure 2 (right) shows the $R_{\mathrm{AA}}$ normalized to the mid-rapidity value [7]. There's very little rapidity dependence until you get to very high $p_{\mathrm{T}}$, where $R_{\mathrm{AA}}$ drops rather sharply with increasing $y$. What could be causing this effect? Well, there are at least two things which are known to vary with rapidity. One is the slope of the jet spectrum, which turns out to be important. For a given energy loss, the steeper the slope, the larger effect you will have on $R_{\mathrm{AA}}$. The other effect is that the quark to gluon ratio increases with rapidity. Quarks are expected to lose less energy than gluons, due to their smaller color factor. These two effects however, go in opposite directions, so it's interesting to look for complementary observables.

For that we can look to the internal structure of jets. What's referred to as the jet fragmentation function is the distribution of the ratio $(z)$ of the hadron $p_{\mathrm{T}}$ to the reconstructed jet $p_{\mathrm{T}}$. Figure 3 shows the ratio of $\mathrm{PbPb}$ to $\mathrm{pp}$, in different bins of centrality [11]. In central collisions there is a departure from pp that consists of an excess of low $z$ fragments, followed by a depletion at intermediate $z$, which one would naturally interpret as energy being transferred to lower momentum. There's also an excess of particles at high $z$ though, which is trickier to understand.

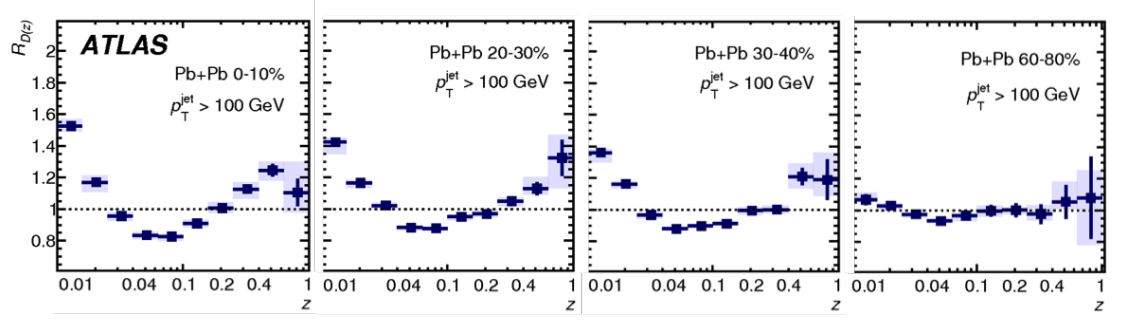

Figure 3: Ratio of jet fragmentation function in $\mathrm{PbPb}$ to $\mathrm{pp}$ collisions for various centrality selections [11].

One model attributes this modification pattern to the difference in radiative energy loss of gluons vs. quarks, due to the difference in the color factor [12]. This model is able to explain both the high and medium $z$ fragmentation as well as the rapidity dependence of $R_{\mathrm{AA}}$ using only a different energy loss for quarks and gluons. Here we have another example of a fragmentationdependent quenching mechanism, but one which is rather different than the picture which arises from JEWEL. Here the quenching depends on the initiating parton rather than evolution of the parton shower in the medium.

Promising new measurements are emerging that look into more detail into the internal structure of jets, but for which the interpretation is still not entirely ironed out. The jet mass is a quantity that is sensitive to the virtuality of the parton that initiates the shower. Quenching is expected to increase the jet mass via momentum exchange with the medium. What's observed is that jet mass distributions in central $\mathrm{PbPb}$ collisions look surprisingly close to PYTHIA, which is a generator that doesn't include quenching [13]. The most economic explanation is that the core of the jet essentially remains intact despite quenching. Models of quenching don't manage to capture this effect. Q-PYTHIA [14] models the entire quenching effect as an increase in the parton splitting, and it clearly overestimates the effect. A long due update to this model is expected soon, however. JEWEL shows a shift to lower mass due to radiative energy loss, but then an overcompensating shift 
to large a mass once the effect of the recoiling medium is included, suggesting that there could be some cancellation of effects at play [15].

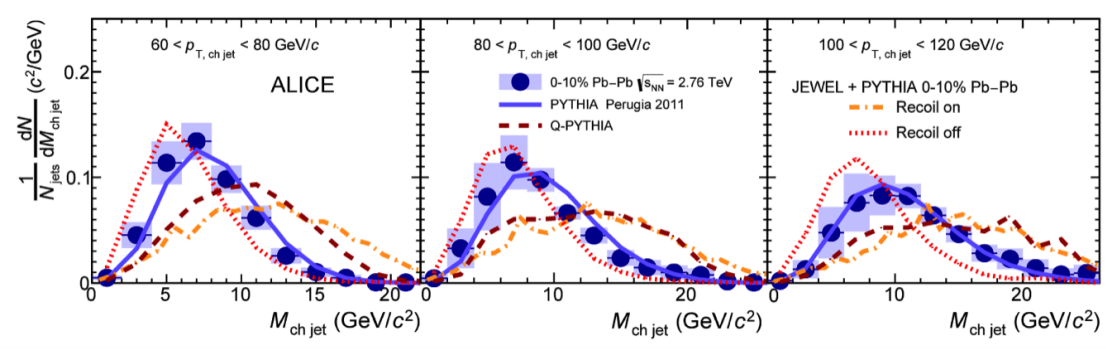

Figure 4: Jet mass distribution for various $p_{\mathrm{T}}$ selections [13].

Another way of looking at the internal structure of jets is to study their substructure. Jet substructure has increasingly come into use in high energy physics primarily to differentiate highly boosted objects from vanilla QCD jets. In a nutshell, the idea is that you can run certain jet algorithms in reverse, which allows you to identify the hardest splittings within the jet. In the context of jet quenching it's particularly interesting to look at how the energy is shared between the two leading subjets, which is quantified by the variable $z_{\mathrm{g}}$. In the pp case, this is directly related to the Alterelli-Parisi splitting function that describes QCD evolution. For quenched jets, we expect this may be modified by coherence effects between nearby partons in the jet [16], an idea which has so far not been directly tested. CMS was able to resolve subjets down to an opening angle of about 0.1. The subjet identification requires a grooming procedure to remove the effect of soft radiation. The so-called soft drop procedure was used for this purpose [17]. Figure 5 shows the $z_{\mathrm{g}}$ distribution in $\mathrm{pp}$ and $\mathrm{PbPb}$ [18], where indeed this distribution is modified as we go to central events. In particular we see relatively fewer symmetric subjets with increasing centrality, which would be consistent with a picture in which the partons are losing energy at least partially independently at the scales being probed. Interestingly, this effect goes away not only in peripheral events but also in for higher $p_{\mathrm{T}}$ jet (not shown). Although it's tempting to interpret this in terms of a modified splitting function for quenched jets, there are a number of other explanations on the market that will need to be confronted. However, I think these recent measurements will prove very useful in understanding the quenching process in much greater detail than we do at present.

\section{Collectively in small systems}

The so-called ridge is a long-range rapidity correlation amongst particles that sits underneath the short-range correlation due to jets. This phenomenon was first observed in AuAu collisions at RHIC [19]. While its origin was not entirely clear at the start, it was recognized that it must arise at early time in order to span such a large range in rapidity. The surprise at the LHC was that this phenomenon also shows up in pA [20] and even in pp [21], at least when you select events with high enough multiplicity. The ridge is now understood to a product of collective effects. Azimuthal flow measures particle production with respect to a reference plane using a Fourier analysis. Due to the almond shape of the overlap region in non-central events, the second harmonic was expected to dominate, meaning there are more particles along the short axis than the long one, due to the 


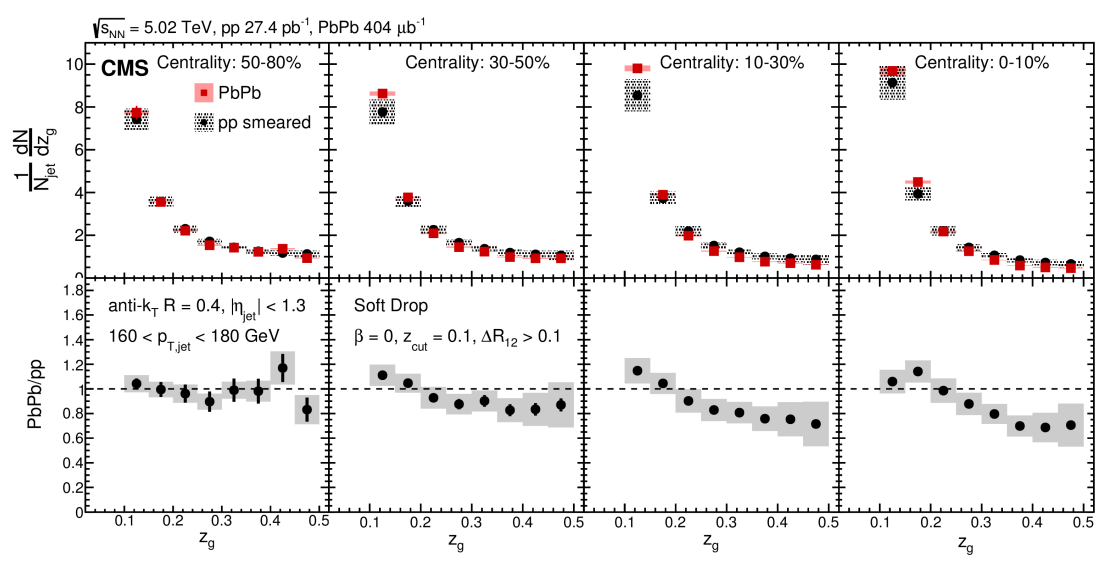

Figure 5: The groomed momentum fraction distributions for various centrality selections of $\mathrm{PbPb}$ collisions and the corresponding ratios to pp collisions [18].

larger pressure gradient. What was not initially recognized was the importance of event-by-event fluctuations in the shape of the overlap region. When these fluctuations were taken into account it was recognized that the ridge arises from the combined contribution of higher order terms.

These higher order terms turn out to be quite useful, which we can understand by analogy with the cosmic microwave background. Variation in the temperature of the CMB reflect the density fluctuations of the early universe before the inflationary period. In much the same way, the azimuthal anisotropy in long range correlations in central heavy ions collisions reflect fluctuations of the initial state of dense QCD matter before its hydrodynamic expansion. While such hydrodynamic calculations provide an accurate description of flow measurements overall, there is some sensitivity to how the initial state is modeled. That in turn effects the estimation of the viscosity, and in fact our knowledge of the initial state is at present the limiting factor in how well we can extract this quantity.

While two-particle correlations were a useful discovery channel for flow effects they suffer from residual correlations from other sources, notably from jets. More robust methods rely on correlations between more than two particles. Figure 6 show $v_{2}$ from correlations between higher numbers of particles in pp, pA and AA, as a function of event multiplicity. The $v_{2}$ rapidly saturates, which suggests that the correlations are really collective in nature. This collectivity is firmly established in all three collision systems once the multiplicity is about 100 particles. This indicates that collectivity is somehow a universal feature, which appears to be related to fluctuations in the source size even at the scale of the proton.

To further confront this picture, one can look at systems that have an intrinsic triangularity, which has been done at RHIC by colliding Au with helium-3 nuclei [23]. It turns out hydrodynamics gives a generally good description of $v_{2}$ and $v_{3}$ in central He $3+\mathrm{Au}$ collisions. This being said, these small systems show a larger sensitivity not only to the initial conditions, but also in the dynamics that occur before equilibrium is established, which is less important for heavier systems.

In pp collisions the extraction of the flow coefficients at the quantitative level turns out to be a rather delicate affair. ATLAS showed that multi-particle correlations can be quite sensitive to certain details of the event classification, as well as to residual non-flow effects [24]. Recently, 


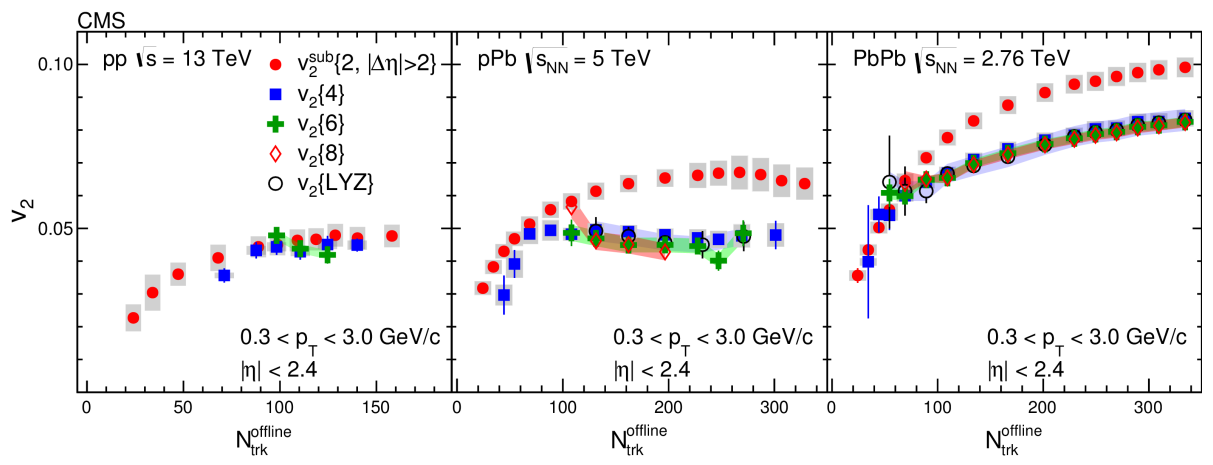

Figure 6: Multiparticle correlations amongst different numbers of particles in pp, pA and AA [22].

however, they introduced a new method where particles are taken from different regions of the event to circumvent these limitations. By doing so they managed to confirm a positive flow signal for multiplicities down to at least 100 particles [25]. The emerging consensus is now that high multiplicity pp collisions do in fact exhibit collectivity. What's still debated is whether hydrodynamics is really valid in such small systems, or whether such effects could result purely from initial state fluctuations without final state effects. These are questions that will have to be resolved in the coming years, but in any case, it's interesting to observe novel effects related to high density QCD, which have so far not been considered in the modeling of these systems.

\section{If not jet quenching, then what?}

Now that we've seen that collective effects are present in small systems, it's natural to ask whether we see effects related to high density QCD on jets. Based on the spectra of jets and charged hadrons in pA, the answer is not obviously yes. The nuclear modification factors don't show any suppression as you would expect for jet quenching. Instead there's even the hint of an enhancement. That's believed to come from modifications of the parton distributions in nuclei (nPDF). These measurements don't have the precision to constrain these effects though.

For that we can turn to dijet measurements. Dijets allow you to constrain the underlying hard scattering kinematics. For LO $2 \rightarrow 2$ scattering, the dijet pseudorapidity goes as the log of the ratio of the Bjorken $x$ in the proton to that in the nucleus. One can also scan in the virtuality $Q$, by varying the $p_{\mathrm{T}}$ of the dijets. Nuclear effects amount to a tiny shift in the $\eta$ distribution. However, if we normalize by the number of dijets, most of the uncertainties on the measurement cancel, allowing one to probe differences of this size. It turns out that by scanning over the $\eta$ distribution we can probe the different regimes of nuclear effects, from shadowing to anti-shadowing to the EMC effect. The dijet $\eta$ shift is shown in different bins of dijet $p_{\mathrm{T}}$ in Fig. 7 (left) [26]. This is the difference of the measurement in $\mathrm{pA}$ and $\mathrm{pp}$, which reduces the sensitivity to the underlying proton PDF. Small but significant deviations from pp are observed in $\mathrm{pA}$. The data are compared to the different parameterizations of nPDF effects on the market, and some are clearly more successful than others at describing the trends.

Since the data appeared, the fits from one group have been updated to include LHC data [27]. In addition to probing a large range in $x$, the data also probe a completely different range in $Q^{2}$ 

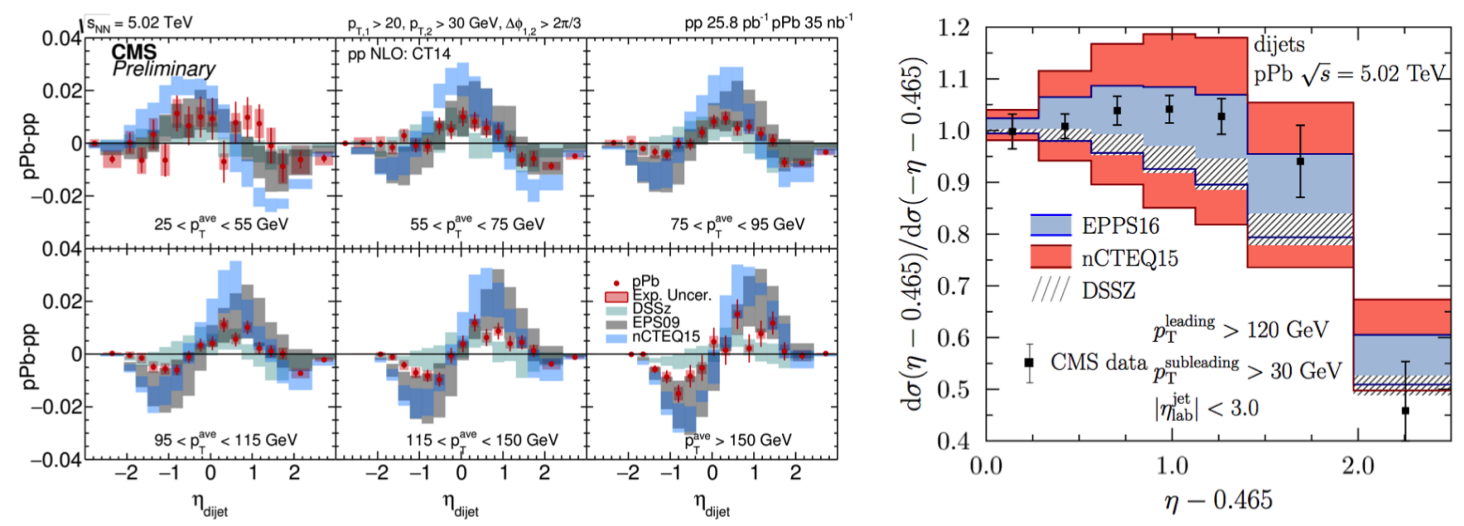

Figure 7: Left: Difference of dijet $\eta$ distributions in $\mathrm{pA}$ and pp, in bins of dijet $p_{\mathrm{T}}$ [26]. Right: Dijet forward-backward asymmetry in pA, compared to $\mathrm{pQCD}$ calculations with various nPDFs [27].

than the previous constraints, which are mainly from DIS and neutrino DIS experiments. Figure 7 (right) shows forward-backward ratio of the pA data. Good agreement is found with previous fits, which is a rather remarkable success of collinear factorization in describing the evolution of nuclear effects over several orders of magnitude in $Q^{2}$. The data give the best constraints so far on the nuclear gluon distribution at intermediate and large Bjorken $x$.

Coming back to the nuclear modification factor, perhaps it's not so surprising we haven't seen quenching effects, since collective effects only show clearly up in high multiplicity collisions. So what happens if we now try to select events according to their activity? Unlike for correlations, this cannot be done by selecting on the multiplicity at mid-rapidity, which is too tightly correlated to the jet production itself. Instead we use the forward energy on the $\mathrm{Pb}$ going side, which should be related to the number of nucleons that participate in the collision. What we observe in Fig. 8 (left) is that $R_{\mathrm{pA}}$ deviates quite strongly from unity, showing a suppression in central collisions and a strong enhancement in peripheral collisions, which is rather unexpected [28].
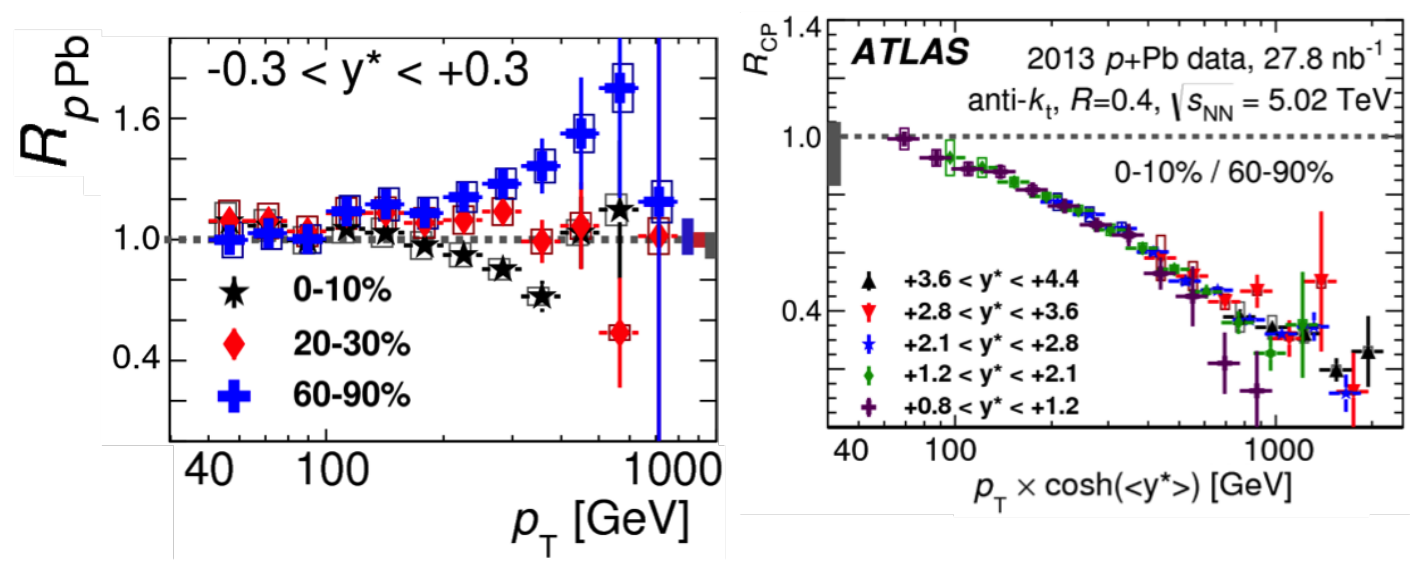

Figure 8: Jet $R_{\mathrm{pA}}$ for several centrality selections (left) and jet $R_{\mathrm{CP}}$ in $\mathrm{pA}$ vs. jet energy on the projectile (proton-going) side (right) [28].

Moreover this deviation is rapidity dependent, showing up most strongly on the proton-going 
side, but with no noticeable effect on the lead-going side. So how can this be understood? A hint emerges if we plot the data vs. the jet energy, rather than $p_{\mathrm{T}}$. The central-to-peripheral ratio for different rapidities on the proton-going side then line up along a common curve, as shown in Fig. 8 (right). The jet energy is proportional to the Bjorken $x$ in the proton, which immediately suggests that this is some kind of energy conservation effect coming from the proton. In other words the more energy you deposit at mid-rapidity, the less is available for at forward rapidity.

This idea can be tested directly in pp collisions using dijets. In Fig. 9 (left) the forward energy is plotted as a function of the mean $p_{\mathrm{T}}$ of dijets, and an anti-correlation is indeed observed, as one would expect from a momentum conservation effect. Moreover, the magnitude of the effect is generally underestimated by event generators. However, there's more to the story, when we look at how the effect depends on dijet rapidity. Again one exploits the fact that the dijets can be related to the parton kinematics. This allows one to plot the energy as a function of $x$ for each of the protons, as shown in Fig. 9 (right). Here we simply define the target side to be the side on which the forward energy is evaluated. It turns out that we only observe a correlation between the forward energy and the proton going in that same direction. This is not what we observe in $\mathrm{pA}$, where we see that the Bjorken $x$ in the proton is correlated to the energy in the backwards direction. Hence, what we observe in pA, doesn't seem to be a trivial energy conservation effect. One idea is that high $x$ protons have a smaller cross section for interaction with the nucleus, suggesting that these protons appear smaller, which may be an explanation, incidentally, for the EMC effect at large Bjorken $x$.
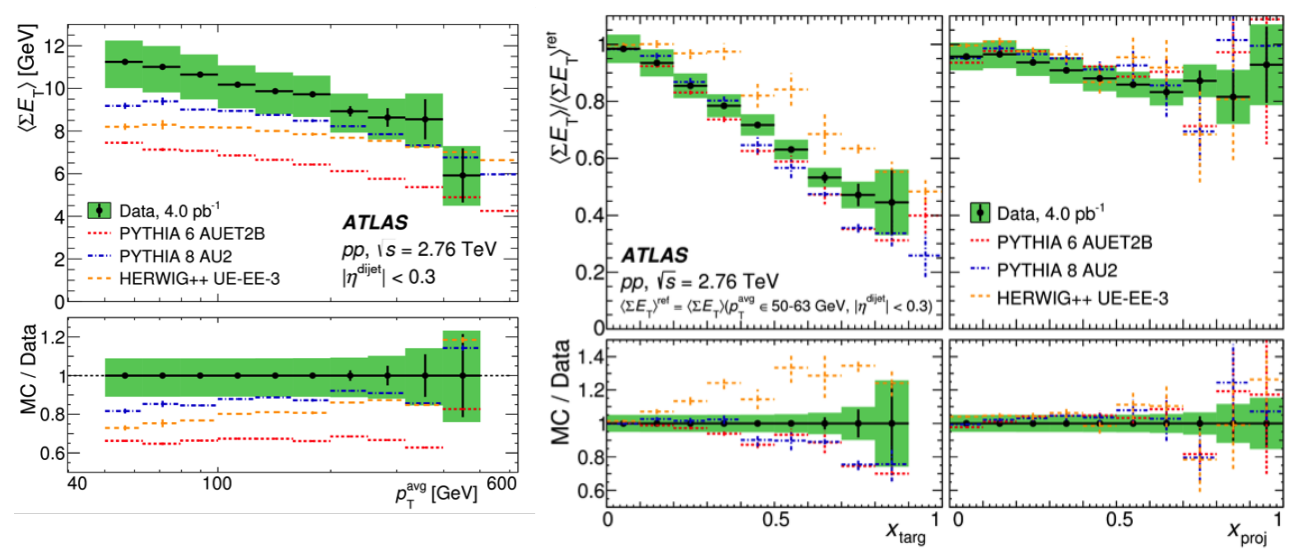

Figure 9: Forward $E_{\mathrm{T}}$ vs. dijet $p_{\mathrm{T}}$ (left) and normalized forward $E_{\mathrm{T}}$ vs. target and projectile $x$ [29].

\section{Conclusions}

Jets and correlations in all collision systems are bringing new insight into QCD in the high density regime. Measurements of jet quenching are increasingly precise, and new measurements of the internal structure of jets are being used to test the relevance of various mechanisms of parton energy loss. Hydrodynamics has been by all accounts very successful in describing AA collisions, and are now reaching the level where we start to be able to extract medium properties with precision. In $\mathrm{pA}$ we have not yet seen any evidence of quenching, but we have managed to further 
constrain nuclear effect on the initial gluon distribution, as well as finding novel effects, while trying to understand the collision centrality. In pp we are certainly benefiting from new techniques such as jet substructure. We also find evidence of collective effects even in high multiplicity pp, although the precise nature and origin of these effects still remain to be elucidated.

\section{References}

[1] PHENIX Collaboration, Suppression of hadrons with large transverse momentum in central $A u+A u$ collisions at $\sqrt{s_{N N}}=130-G e V$, Phys. Rev. Lett. 88 (2002) 022301, [nucl-ex/ 0109003 ].

[2] PHENIX Collaboration, Absence of suppression in particle production at large transverse momentum in $\sqrt{s_{N N}}=200 \mathrm{GeV} d+$ Au collisions, Phys. Rev. Lett. 91 (2003) 072303, [nucl-ex/0306021].

[3] PHENIX Collaboration, Formation of dense partonic matter in relativistic nucleus-nucleus collisions at RHIC: Experimental evaluation by the PHENIX Collaboration, Nucl. Phys. A757 (2005) 184-283, [nucl-ex/0410003].

[4] STAR Collaboration, Experimental and theoretical challenges in the search for the quark gluon plasma: The STAR Collaboration's critical assessment of the evidence from RHIC collisions, Nucl. Phys. A 757 (2005) 102-183, [nucl-ex/ 0501009 ].

[5] D. T. Son and A. O. Starinets, Viscosity, Black Holes, and Quantum Field Theory, Ann. Rev. Nucl. Part. Sci. 57 (2007) 95-118, [arXiv: 0704 . 0240].

[6] CMS Collaboration, Charged-particle nuclear modification factors in PbPb and pPb collisions at $\sqrt{s_{\mathrm{NN}}}=5.02 \mathrm{TeV}$, JHEP 04 (2017) 039, [arXiv:1611.01664].

[7] ATLAS Collaboration, Study of inclusive jet yields in $P b+P b$ collisions at $\sqrt{s_{N N}}=5.02 \mathrm{TeV}$, ATLAS-CONF-2017-009, (2017), https://cds.cern.ch/record/2244820.

[8] J. G. Milhano and K. C. Zapp, Origins of the di-jet asymmetry in heavy ion collisions, Eur. Phys. J. C 76 (2016) 288, [arXiv:1512.08107].

[9] CMS Collaboration, Jet momentum dependence of jet quenching in PbPb collisions at $\sqrt{s_{N N}}=2.76$ TeV, Phys. Lett. B 712 (2012) 176-197, [arXiv:1202.5022].

[10] ATLAS Collaboration, Measurement of jet $p_{\mathrm{T}}$ correlations in $P b+P b$ and $p p$ collisions at $\sqrt{s_{\mathrm{NN}}}=2.76 \mathrm{TeV}$ with the ATLAS detector, arXiv:1706.09363.

[11] ATLAS Collaboration, Measurement of jet fragmentation in $P b+P b$ and pp collisions at $\sqrt{s_{\mathrm{NN}}}=2.76 \mathrm{TeV}$ with the ATLAS detector at the LHC, Eur. Phys. J. C 77 (2017) 379, [arXiv:1702.00674].

[12] M. Spousta and B. Cole, Interpreting single jet measurements in Pb + Pb collisions at the LHC, Eur. Phys. J. C 76 (2016) 50, [arXiv: 1504 .05169].

[13] ALICE Collaboration, First measurement of jet mass in Pb-Pb and $p$-Pb collisions at the LHC, arXiv:1702.00804.

[14] N. Armesto, L. Cunqueiro and C. A. Salgado, Q-PYTHIA: A Medium-modified implementation of final state radiation, Eur. Phys. J. C 63 (2009) 679-690, [arXiv: 0907.1014 ].

[15] R. Kunnawalkam Elayavalli and K. C. Zapp, Medium response in JEWEL and its impact on jet shape observables in heavy ion collisions, JHEP 07 (2017) 141, [arXiv: 1707. 01539].

[16] J. Casalderrey-Solana, Y. Mehtar-Tani, C. A. Salgado and K. Tywoniuk, New picture of jet quenching dictated by color coherence, Phys. Lett. B 725 (2013) 357-360, [arXiv: 1210 . 7765]. 
[17] A. J. Larkoski, S. Marzani, G. Soyez and J. Thaler, Soft Drop, JHEP 05 (2014) 146, [arXiv:1402.2657].

[18] CMS Collaboration, Measurement of the splitting function in pp and PbPb collisions at $\sqrt{S_{\mathrm{NN}}}=5.02$ TeV, arXiv:1708.09429.

[19] STAR Collaboration, Long range rapidity correlations and jet production in high energy nuclear collisions, Phys. Rev. C 80 (2009) 064912, [arXiv: 0909.0191 ].

[20] CMS Collaboration, Observation of long-range near-side angular correlations in proton-lead collisions at the LHC, Phys. Lett. B 718 (2013) 795-814, [arXiv: 1210 . 5482].

[21] CMS Collaboration, Observation of Long-Range Near-Side Angular Correlations in Proton-Proton Collisions at the LHC, JHEP 09 (2010) 091, [arXiv: 1009 . 4122].

[22] CMS Collaboration, Evidence for collectivity in pp collisions at the LHC, Phys. Lett. B 765 (2017) 193-220, [arXiv:1606.06198].

[23] PHENIX Collaboration, Measurements of elliptic and triangular flow in high-multiplicity ${ }^{3} \mathrm{He}+\mathrm{Au}$ collisions at $\sqrt{s_{N N}}=200 \mathrm{GeV}$, Phys. Rev. Lett. 115 (2015) 142301, [arXiv: 1507.06273 ].

[24] ATLAS Collaboration, Measurement of multi-particle azimuthal correlations in $p p, p+P b$ and low-multiplicity Pb+Pb collisions with the ATLAS detector, Eur. Phys. J. C 77 (2017) 428, [arXiv:1705.04176].

[25] ATLAS Collaboration, Measurement of multi-particle azimuthal correlations with the subevent cumulant method in pp and $p+P b$ collisions with the ATLAS detector at the LHC, arXiv:1708.03559.

[26] CMS Collaboration, Dijet pseudorapidity in pp and pPb collisions at $\sqrt{s_{\mathrm{NN}}}=5.02 \mathrm{TeV}$ with the CMS detector, CMS-PAS-HIN-16-003, (2016), https://cds.cern.ch/record/2201538.

[27] K. J. Eskola, P. Paakkinen, H. Paukkunen and C. A. Salgado, EPPS16: Nuclear parton distributions with LHC data, Eur. Phys. J. C 77 (2017) 163, [arXiv:1612.05741].

[28] ATLAS Collaboration, Centrality and rapidity dependence of inclusive jet production in $\sqrt{s_{\mathrm{NN}}}=5.02 \mathrm{TeV}$ proton-lead collisions with the ATLAS detector, Phys. Lett. B 748 (2015) 392, [arXiv:1412.4092].

[29] ATLAS Collaboration, Measurement of the dependence of transverse energy production at large pseudorapidity on the hard-scattering kinematics of proton-proton collisions at $\sqrt{s}=2.76 \mathrm{TeV}$ with ATLAS, Phys. Lett. B 756 (2016) 10-28, [arXiv: 1512.00197]. 